\title{
Cooperative regulation and trading of emissions using plug-in hybrid vehicles
}

October 22, 2012

Arieh Schlote, Florian Häusler, Thomas Hecker, Astrid Bergmann, Emanuele Crisostomi, Ilja Radusch, Robert Shorten

\begin{abstract}
We present a new approach to regulating traffic related pollution in urban environments by utilising hybrid vehicles. We give a number of different strategies and some variants of how to achieve this. The efficacy of our approach is exemplified both by the construction of a proof of concept vehicle and by extensive simulations.
\end{abstract}

\section{Motivation}

Within the context of smart-cities research [1,2], collaborative mobility is rapidly becoming one of the main growth areas in applied and theoretical research. The topic is generating interest in a wide set of areas such as: computer science; electrical engineering; applied mathematics; operations research; networking, as well as transportation engineering. Motivated by the fact that road networks in our cities are often hugely inefficient, and by recent advances in technologies such as communication networks, vehicles, optimization, as well as a proliferation in smart-phones and changing social attitudes to mobility, the demand for services and products related to smart mobility is expected to become a multi-billion dollar industry over the next decade [3]. Examples of successful collaborative mobility products include smart parking systems [4], car sharing products such as car2go in Berlin [6], and other similar products [7].

Collaborative mobility is not a new topic in transportation research. The notion of a vehicle communicating with other vehicles and with infrastructure (V2X) to improve the quality of service (QoS) to the vehicle owner, is closely related to the area of transport telematics [5]. Transport telematics has been an active area of research for several decades but its impact has always been limited by available technologies. However, due to the aforementioned advances, and a degree of convergence in several disciplines, and in particular due to strict new guidelines coming from a diverse set of regulatory bodies, the interest in 
collaborative mobility has never been greater (nor has its potential to deliver improvements in mobility concepts with real societal value). Roughly speaking, transport related regulation is driven by four main factors; congestion on our roads (and related inefficiencies); vehicular safety; greenhouse gas emissions; and the quality of air in our cities. Each of these issues makes a compelling case for investing in smarter transportation systems. Congestion is not only unpleasant to experience, but it is also a major inhibiter of economic growth [8]. Similarly, car-related safety issues, both for occupants and other road users, is a major issue with car accidents still accounting for a disproportionately high percentage of injury related mortality figures [9]. Also, the contribution of transportation to greenhouse gas generation is not sustainable at its current levels [10]; in the EU, transportation accounts for $20 \%$ of greenhouse gas emissions. However, perhaps the most compelling reason to revisit our concept of personal mobility, is the fact that the internal combustion engine (ICE) is extremely damaging to human health [11]. By-products of the ICE include: $C O ; N o X ; S O ; O z o n e$; Benzine; PM10; and PM25. All of these affect humans adversely and are linked to lung disease, heart disease, and certain cancers. In a recent study in the US [12] it is claimed that problems with air-quality lead to three times as many deaths as car accidents. Amazingly, just how damaging to health those vehicles can be, appears not to be widely appreciated by the general public with public discourse focussing mainly on greenhouse emissions and on vehicle safety. This basic fact provides the main motivation for this and prior work [13, 14]; namely, to investigate methods based on V2X to contribute to the regulation of air quality in our cities.

Before proceeding, it is worth noting that governments and municipal authorities have already started to respond to the air-quality issue. Car manufacturers are under constant pressure to produce ever-cleaner vehicles. Further, cities in some countries ban certain vehicles from densely populated areas (Umweltzonen) [15], and sometimes speed limits are adapted to respond to pollution peaks [16]. Going further in this direction, strict measures are being planned to ban the ICE from our cities in the near future [17]. However, even though these measures are very welcome, they do not go far enough. Roughly speaking, they suffer from three main drawbacks.

(i) First, they are per-vehicle measures. Degradation of air quality results from the aggregate effect of vehicles. Enforcing per-vehicle measures (unless we ban vehicles all together), takes no account of this effect. In fact, while the per-car emissions have been successfully decreased in the last years, the growth in the number of new cars has led to a substantial effective increase in the overall (aggregate) emission output in certain regions [8].

(ii) These measures are open-loop measures. The regulation is the same irrespective if there is one vehicle in a spatial area (in the middle of the night), or if there are millions of vehicles in the same area. Why not allow 
one slightly dirty vehicle into an Umweltzone if it is the only vehicle in the zone? Also, cars can respond to external pollution sources (if the air is dirty then cars can be cleaner). This degree of freedom has only be explored in a limited manner.

(iii) All of these measures are highly invasive and affect the vehicle owners in a very disruptive manner. They may even have unintended consequences for the city. Forcing vehicles away from an certain zone may lead to congestion elsewhere or even a higher total amount of emissions in the whole city.

Our objective in this paper is to present a method to regulate pollution without overly inconveniencing the vehicle owner. Essentially, we wish, based on estimates of pollution levels, to create a feedback loop that allows vehicles to change their behaviour so as to keep pollution below a safe pre-described level. Traditionally, this can be achieved by adapting speed limits, re-routing vehicles, and by changing traffic light sequencing. These measures are highly invasive. However, new vehicle types, such as electric vehicles, and plug-in hybrid vehicles, allow us new and exciting degrees of freedom in addressing the pollution regulation problem, potentially, without inconveniencing the vehicle owner at all. The key idea here is to view a battery as a type of filter for vehicular traffic that separates the location where energy is used and the location where it is being produced (perhaps using fossil fuels or other "dirty" forms of energy). Essentially we generate the energy in a place that is away from humans, and centrally, so that pollutants can be filtered, gathered and neutralised, and we deliver it, via batteries, in a form close to humans that is clean and safe. When taking this point of view, new vehicle classes such as electric and hybrid electric vehicles that allow cars to traverse sensitive areas without polluting them, become a powerful tool in controlling pollution levels in cities. Power-split hybrid vehicles, in particular, which can be operated in fully electric, and in ICE mode, allow us to control the manner in which pollution is delivered into the environment. Thus by orchestrating the way in which a fleet of such vehicles switch into fully electric mode (based on a function of the aggregate pollution levels), one should, in principle, be able to regulate pollution levels in a manner that is non-invasive to the driver, and is highly flexible. The vehicle only uses as much electric power as is necessary to keep the aggregate pollution level below a certain threshold. It is the main contribution of this paper to show that this is indeed the case. To do this we construct a proof-of-concept context aware hybrid vehicle: the twinLIN. We then show via simulation that by coordinating the behaviour of a fleet of such vehicles pollution levels can be regulated in an efficacious manner, with minimal vehicle-to-vehicle and vehicle-to-infrastrucure (V2X) communication requirements. This latter objective is achieved by borrowing and developing ideas from the design of packet routers, that allows us to realise a notion of best effort behaviour in transportation networks. Finally, we describe a suite of related applications that can be developed based on the platform described in this paper. 
This article is organised as follows: In Section 2 we present some important notions from networking research and describe how these translate in a seamless fashion to fleets of hybrid vehicles. In Section 3.1 we describe our hybrid car and the modifications we made to it as well as the smart phone application we used. The pollution model we used is given in Section 3.2. Section 3.3 outlines a number of employed control algorithms. Simulation results are presented in Section 4. In Section 5 we conclude this paper by outlining related applications that can be developed from our basic ideas and how our ideas may be applied to if only conventional combustion driven cars are available.

\section{An analogy with Internet congestion control}

The principal objective of our paper is to use the opportunity afforded by V2X, and by new vehicle types, to develop effective techniques to regulate pollution generated by road vehicles. Essentially we wish to place a feedback loop around a group of vehicles, and use this loop to control the group emissions. Our basic requirements are as follows.

(i) Aggregate pollution levels should not exceed pre-described levels : Basically the objective here is to decouple pollution in a certain area (or group of vehicles) from the number of vehicles in that area as depicted in Figure 1.

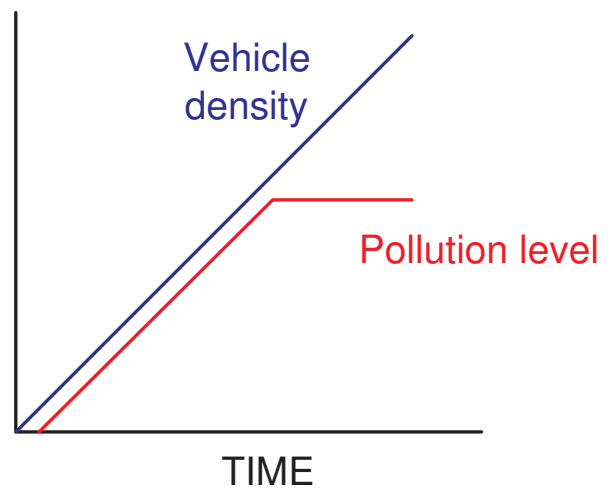

Figure 1: Typically pollution levels follow the number of vehicles. We wish to decouple pollution from vehicle density.

(ii) Best effort behaviour : The network traffic should exhibit a best effort type behaviour. Vehicles in a geographic area should adjust their behaviour so as to share their the allowed pollution level irrespective of the number of participating vehicles. They should also respond to non-vehicle pollution generation by becoming cleaner if pollution levels rise. 
(iii) Fairness : Ideally, we would like to see a notion of fairness in the network. Cars that are more polluting should be more inconvenienced than less polluting ones.

Each participating vehicle should strive to achieve these objectives by using as little battery power as possible. Electric power is considered very valuable as it takes a long time to charge these vehicles, and zero-emission power gives these vehicles access to certain restricted areas of the city. One important other point to note is that we do not require zero pollution in a geographic area. Such a constraint imposes a high burden on participating vehicles. Rather, we impose a pollution budget and insist, that the aggregate level of pollution, among all participating vehicles, never exceeds this level.

Items (i)-(iii) resemble closely the requirements of resource allocation algorithms found in networking applications (such as the Internet). The notion of aggregate behaviour, best effort behaviour, and fairness, resemble in particular elastic traffic control in the Internet as depicted in Figure 2. Essentially, we are viewing the pollution control problem as a resource allocation problem, where a certain amount of pollution is shared among competing vehicles. In our situ-

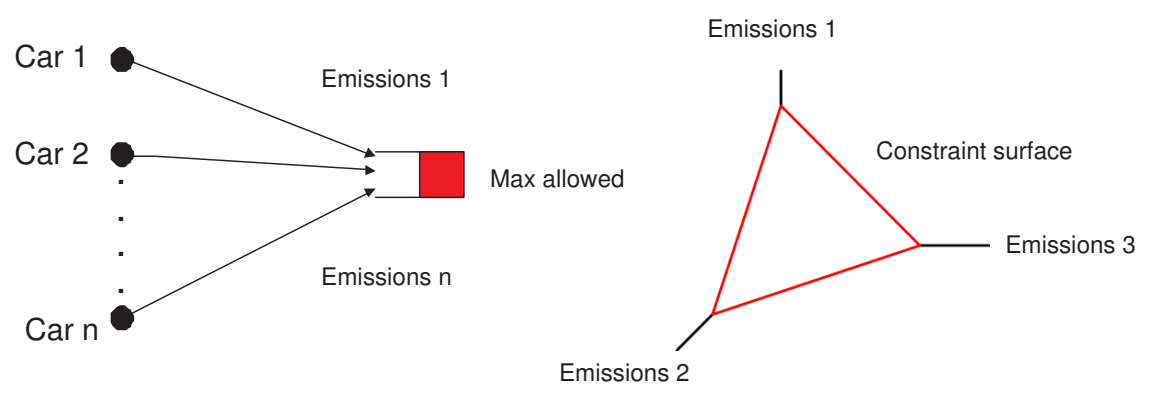

Figure 2: Pollution as a resource allocation problem

ation the objective of each vehicle is to be as polluting as possible (use as little battery power as possible) while at the same time being fair to other vehicles, and such that the aggregate level is close to the allowed threshold. Note also, that we would like vehicles to change the behaviour to respond to vehicles joining and leaving the network (best effort behaviour).

The realisation that the pollution control problem can be recast in a resource allocation framework is fortunate. Network resource allocation problems are at a mature stage, and typically involve a large scale decentralised optimization problem. This latter feature makes algorithms from this community attractive in a transportation context. Relevant ideas in this direction include the Kelly 
framework [20], RED [19, 27], AIMD congestion control [18], to name but a few. In the next section we shall illustrate how some of these ideas, together with new vehicle types, can be used to great effect to manage aggregate vehicle emissions.

\section{Pollution control}

As mentioned in the previous section we wish to use inter vehicle cooperation to regulate pollution in a manner that is somehow invisible to the driver. Our approach to achieve this is to use hybrid vehicles as a demonstration platform. The basic idea is to orchestrate and coordinate switching between drive modes in a fleet of hybrid vehicles so as to achieve regulated pollution levels. Feedback is used to adjust the level of coordination to achieve the desired level of regulation. The control loop is depicted in Figure 3. To realise this objective we assume

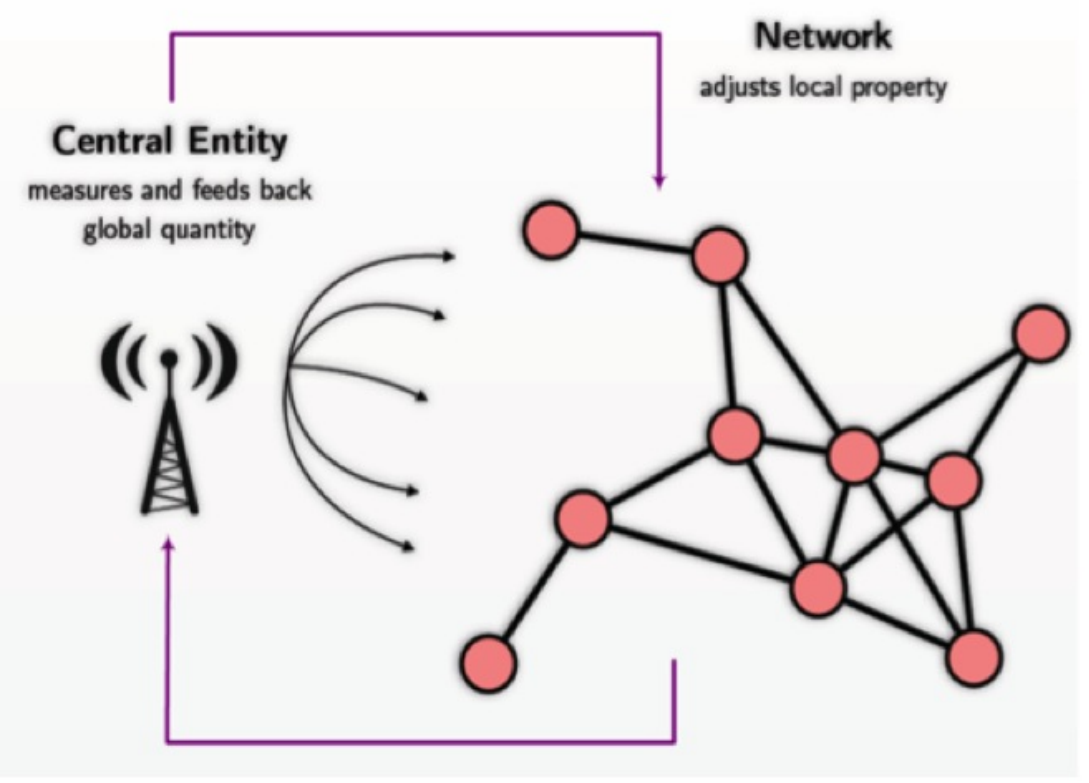

Figure 3: Feedback loop

the availability of a context aware hybrid vehicle, whose switching into fully electric mode can be made dependent on the location of the vehicle as well as in response to an external signal. We now describe one such vehicle, which we have constructed, and then proceed to outline our basic modeling assumptions, and control strategies. 


\subsection{The networked twinLIN car}

To demonstrate the application of our approach we equipped a 2008 model Toyota Prius with technology. The car is depicted in Figure 4. The Toyota

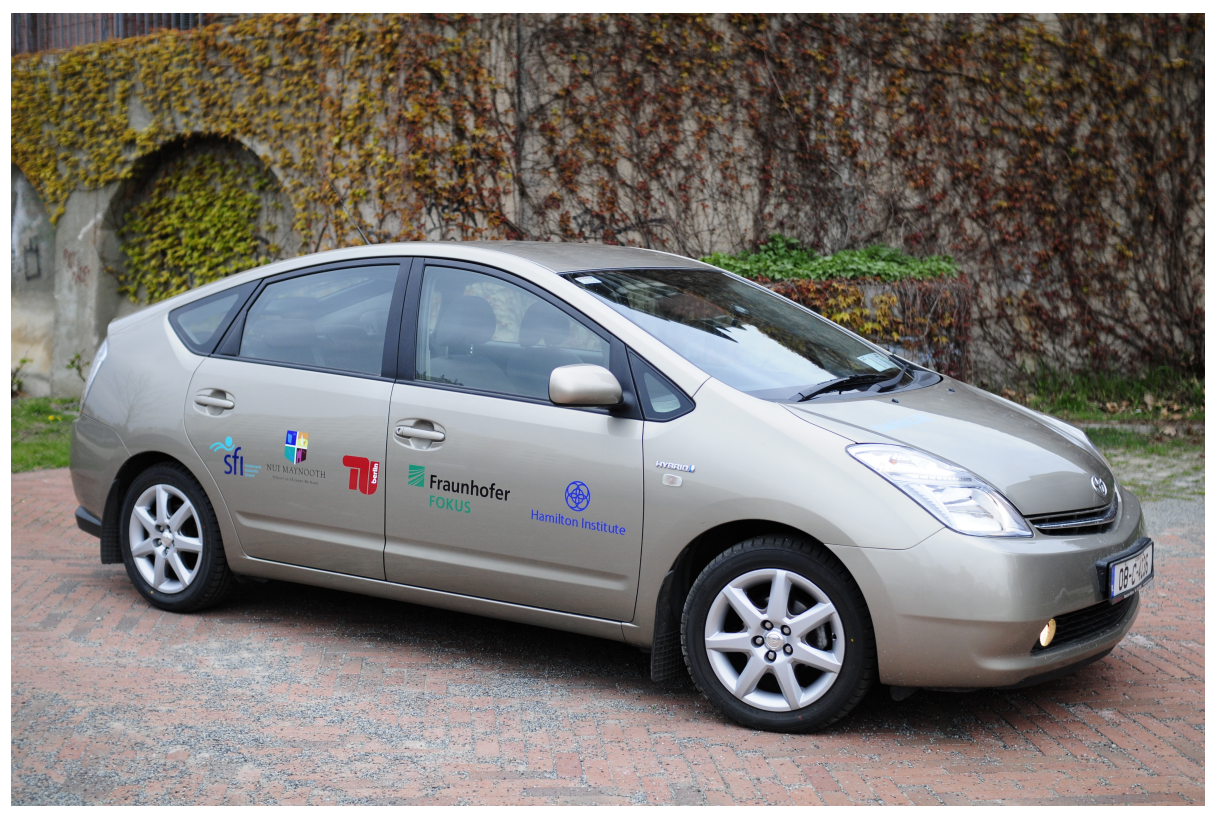

Figure 4: twinLIN

Prius is a power split hybrid vehicle [21]. The car can operate in fully electric mode, in ICE mode, or in combined mode. The power split device also allows the car to function as a series hybrid where the gasoline engine can operate to charge the batteries. The driving mode is determined by the vehicle control unit in normal operation. The Prius is equipped with an "EV-mode button" allowing the vehicle to be manually switched to pure electric driving mode if the battery level permits. This mode is automatically turned off if the button is pushed again; when the vehicle speed rises above $45 \mathrm{~km} / \mathrm{h}$; or when more torque is required than the electric motor can deliver (or when the battery level is too low). For the purpose of this project we have made some important modifications to the basic vehicle to make it behave as a context aware vehicle.

First, special purpose hardware was constructed to allow communication between a GPS device and other vehicles, and to control switching between the various engine modes. Communication to other vehicles and to GPS is realised using an Android smart phone device. For our work a SAMSUNG NEXUS device and HTC EVO-3D were used, both running Android 4.0. Communication to other vehicles and to a central (cloud) server is via the cellular network, and communication from the phone to other systems in the vehicle is via bluetooth. To control the driving mode, the software connects via bluetooth to a propri- 

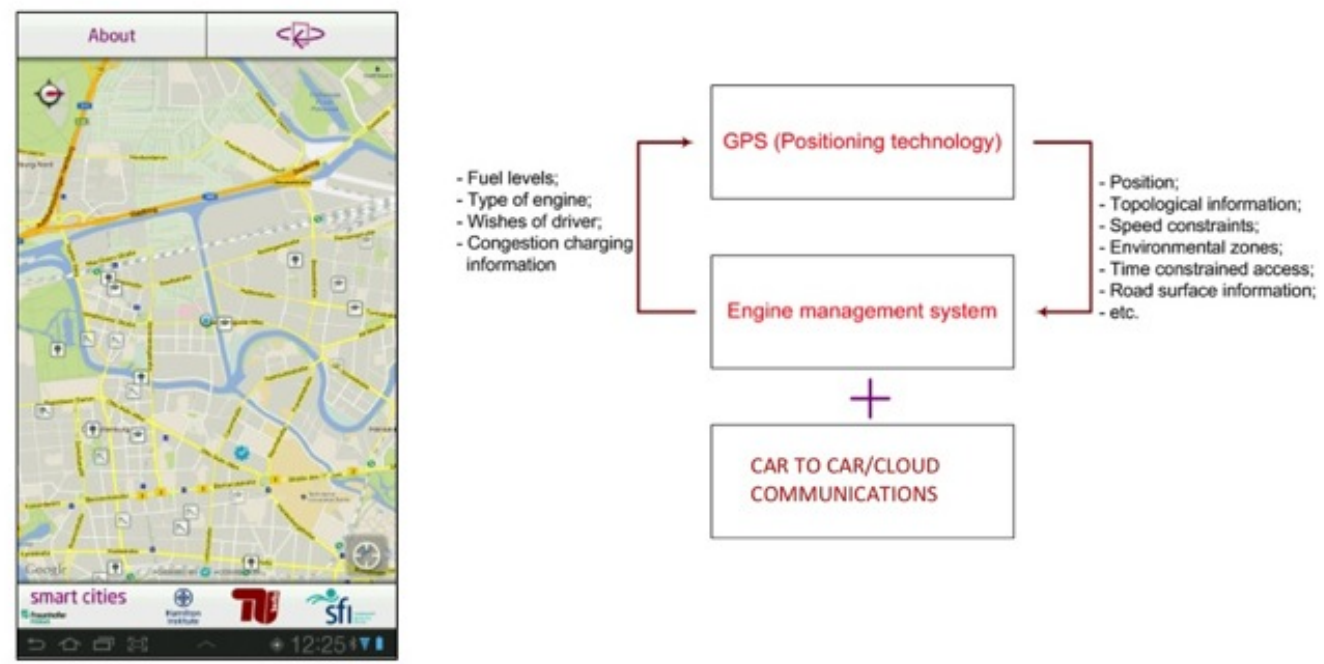

Figure 5: Vehicle communication paths. Communication paths internally in the vehicle and to other vehicles and infrastructure is achieved using an Android application platform.

etary On-board diagnostics II (OBD-II) interface to the vehicle's controller area network (CAN) bus, and to a mechanical switch to control switching between the EV mode and non-EV driving modes. Thus, the smartphone has access to in-vehicle bus data as driving mode, battery level, and pollution levels via a central server. A screenshot of the smartphone App is depicted in Figure 5. The vehicle and the Android application thus allows the vehicle to interact with its environment in a very smart manner. Importantly, it allows controlled delivery of emissions and pollution into the city environment (be it noise pollution, or more directly harmful pollutants), allowing us to control where and when these emissions are delivered into the environment. A movie demonstrating operation of the vehicle can be found at http://www.hamilton.ie/aschlote/twinLIN.mov. 


\subsection{Pollution modeling and simulation}

To investigate the cooperative behaviour of a fleet of twinLIN-like hybrid vehicles, we use a modified version of the traffic simulator SUMO (Simulation of Urban MObility) [26]. SUMO is an open source microscopic traffic simulator [23] that was developed at the Institute of Transportation Systems at the German Aerospace Center, and is licensed under the GPL. Sumo is used to simulate real traffic flows for a mix of hybrid vehicles traversing a specified geographic location. Average speed emission models are used to estimate specific pollutants of each vehicle $[22,24,25,35]$. The average-speed approach is described in detail in the UK Design Manual for Roads and Bridges (DMRB) [22] and the European Environment Agency's COPERT model [25]. According to these average-speed models, the emission factor $\mathrm{f}(\mathrm{t}, \mathrm{p})$ in grams per kilometer is computed as:

$$
f(t, p)=\frac{k}{v}\left(a+b v+c v^{3}+d v^{3}+e v^{4}+f v^{5}+g v^{6}\right)
$$

where $t$ denotes the type of vehicle (and depends on fuel, emission standard, category of vehicle, engine power), $p$ denotes the particular type of pollution of interest (e.g. CO, CO2, NOx, Benzene), $v$ denotes the average speed of the

vehicle, and the parameters $a, b, c, d, e, f, g$ and $k$ depend on both the type of vehicle and the pollutant $p$ under consideration. For the purpose of this work, the values of the parameters are taken from Appendix D, in reference [24]. In Equation (7) it is assumed that speeds are measured in $\mathrm{km} / \mathrm{h}$ and emission factors in $\mathrm{g} / \mathrm{km}$. At this point it is probably worth noting that the averagespeed model suffers from the drawback that very different vehicle operational behaviours are characterized by the same average speed. We use the average speed model here for simplicity. More realistic and accurate models can easily be embedded into the simulation environment without changing the qualitative features of the simulation (or the analysis). As we shall see, the key point in our work is the assumption that the pollution levels in the group are an increasing function of the number of vehicles in fully EV mode.

\subsection{Control algorithms}

In this paper we investigate three control methods for regulating pollution in geographic area by orchestrating the switching of vehicles into fully electric mode. Our first controller is a classical proportional-integral-differential (PID) regulator. This control is based on the following assumptions.

(i) Denote by $E(k)$ the amount of pollution produced by all vehicles in a geographic area at time instant $k$. Let $p(k)$ be the probability that an individual vehicle is using its combustion engine at time $k$. We assume that there is an increasing, possibly non-linear relationship between the average levels of of pollution $\bar{E}(k)$ generated by the vehicles (in a geographic area), and the value of the signal $p(k)$.

(ii) Pollution levels in a geographic area can be measured or estimated without explicit communication from the vehicles. 
(iii) There is no explicit car to car communication.

(iv) There is no explicit car to infrastructure information, but participating vehicles can listen and respond to broadcast measurements.

PID control : With these assumptions we can set up the regulation problem classically as depicted in Figure 3 where $E^{*}(k)$ denotes the desired level of pollution at time instant $k$. The first control strategy uses a discrete implemen-

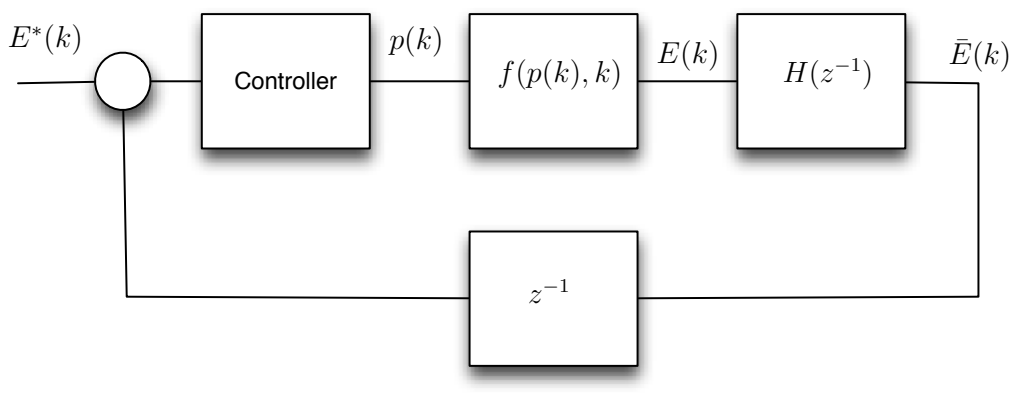

Figure 6: Basic control loop. The first control strategy uses a discrete implementation of the classical PID control.

tation of the classical PID control:

$$
p(t)=K_{p} e(t)+K_{i} \int_{0}^{t} e(t)+K_{d} \frac{d e(t)}{d t}
$$

where $e(t)=E^{*}(t)-E(t)$. The plant to be controlled is modeled as a sectorbounded time-varying nonlinearity with additive noise term (to account for stochastics), followed by an averaging filter. Note that the nonlinear term depends on traffic densities and is thus time-varying. Given this background, the problem can viewed as a classical Lure problem and the control design carried out in this framework [30, 29]. Consequently, stability of the closed loop can be guaranteed by selecting the control gains in accordance with the Circle Criterion. A detailed description of a design using the circle criterion for a similar 
problem is given in [29]. In this paper, a similar model is derived in the context of a buffer length regulation problem for a network router and the circle criterion in employed to give a stable controller. We shall not repeat this description here. Rather, it is worth noting that even though the Lure framework allows filter dynamics to be incorporated into the feedback loop (this is an advantage over other approaches to nonlinear control designs such as multiple-increase, multiple-decrease algorithms [28]), the modeling of the process is not satisfactory. The system to be controlled is a stochastic one; the Lure approach models this as a deterministic system with an additive noise term and in some sense ignores the stochastic nature of the problem. In what follows we give an exact analysis in a stochastic framework for a simple integral control. A similar approach can be taken to obtain the same results if we use a PID controller.

To this end we further assume that desired pollution level $E^{*}(k)=E^{*}$ is the same at each time step $k$. Also we assume that the relationship between $p(k)$ and the expected value of $E(k+1)$ is linear. For a fixed number of cars, let the emissions of car $i$ at time $k$ be given by

$$
E_{i}(k)=\left\{\begin{array}{lll}
C_{i} & , \text { with probability } & p(k), \\
0 & , \text { with probability } & 1-p(k),
\end{array}\right.
$$

for some $C_{i} \geq 0$. The overall emissions at time $k$ are given by $E(k)=\sum_{i} E_{i}(k)$. We now analyse this system for our integral controller, where we use a filtered value for the emission measurements given by $\bar{E}(k+1)=\lambda E(k+1)+(1-\lambda) \bar{E}(k)$ and update the probability according to

$$
p(k+1)=Q\left(p(k)+K\left(E^{*}-\bar{E}(k)\right)\right),
$$

where $C \geq E^{*}>0$ is the desired pollution level and $C=\sum_{i} C_{i}$ is the maximum possible pollution level and $K>0$ is the gain. To ensure that $p(k) \in$ $[0,1]$ we use $Q: \mathbb{R} \rightarrow[0,1]$, a projection to the unit interval, i.e

$$
Q(p)=\left\{\begin{array}{lll}
0 & \text { if } & p<0 \\
p & \text { if } & 0 \leq p \leq 1 \\
1 & \text { if } & 1<p
\end{array}\right.
$$

We now focus our attention on the expected values of $\bar{E}$ and $p$ conditioned on the initial values $\bar{E}(0)$ and $p(0)$. If we let $X(k)=E[\bar{E}(k)]$ and $Y(k)=E[p(k)]$, then if $Y(k)$ is far enough from the boundary of the unit interval for all $k \in \mathbb{N}$ we obtain the relationships

$$
\begin{aligned}
& X(k+1)=\lambda C Y(k)+(1-\lambda) X(k) \\
& Y(k+1)=Y(k)+K\left(E^{*}-X(k)\right) .
\end{aligned}
$$

Theorem 1. The system described by Equations (6) and (7) has a unique fixed point, which is independent of the initial conditions. If further $K<\frac{1}{C}$ then the fixed point is locally asymptotically stable. 
Proof. We can describe the system in matrix notation as

$$
\left(\begin{array}{c}
X(k+1) \\
Y(k+1)
\end{array}\right)=P\left(\begin{array}{c}
X(k) \\
Y(k)
\end{array}\right)+W
$$

wih $P=\left(\begin{array}{cc}1-\lambda & \lambda C \\ -K & 1\end{array}\right)$ and $W=\left(\begin{array}{c}0 \\ K E^{*}\end{array}\right)$ The fixed point is given by $\left(\begin{array}{c}E^{*} \\ \frac{E^{*}}{C}\end{array}\right)$. It can be seen that under the assumption that $K<\frac{1}{C}$ the spectral radius of $P$ is less then 1 . And hence the system described by Equation (8) converges locally exponentially fast to its asymptotic value.

Random Early Detection: RED As an alternative to the PID we also investigated a feedback strategy that emulates the RED algorithm used in internet congestion control. RED is the acronym for Random Early Detection [38]. The basic idea in RED is to adjust the probability of a car switching into fully electric mode as a non-linear function of the average pollution levels $\bar{E}$. The basic idea is depicted in Figure 7. The control objective in RED is to maintain pollution levels between pre described thresholds: $E_{\min }$ and $E_{\max }$. To do this one describes a probability curve (also depicted in Figure 7)

$$
p(k)=f(\bar{E}(k)) .
$$

Basically, the hope is that for a range of traffic densities there exists equilibrium probabilities $p^{*}$ corresponding to $E^{*}$ such that $E_{\min } \leq E^{*} \leq E_{\max }$, and that these equilibria are stable so that the system converges.

The stability of RED has been widely studied in the context of internet congestion control [19]. See for example [27], where RED is emulated in a distributed fashion. We will not repeat these stability discussions here. Rather we concentrate on the merits of RED, or otherwise, in the present context. Clearly, RED is much simpler than the PID control described above. It requires only a non-linear function for implementation. However, it is also clear that the probability curve is predicated on a range of vehicle densities. Not all densities can be accommodated using a single curve, and to do this would require adjustment of $f$ based on feedback. There are approaches to do this, see for example [37]. A further concern is the performance of the algorithm in the presence of rapidly varying traffic densities.

Multiplicative Increase Multiplicative Decrease: MIMD As a further alternative control algorithm we investigated MIMD. Here again the goal is to keep the filtered emissions $\bar{E}(k), k=0,1, \ldots$ within some interval $\left[E_{\min }, E_{\max }\right]$. To this end two factors $m$ and $M$ satisfying $0<m<1<M$ are choosen and the probability is updated according to 

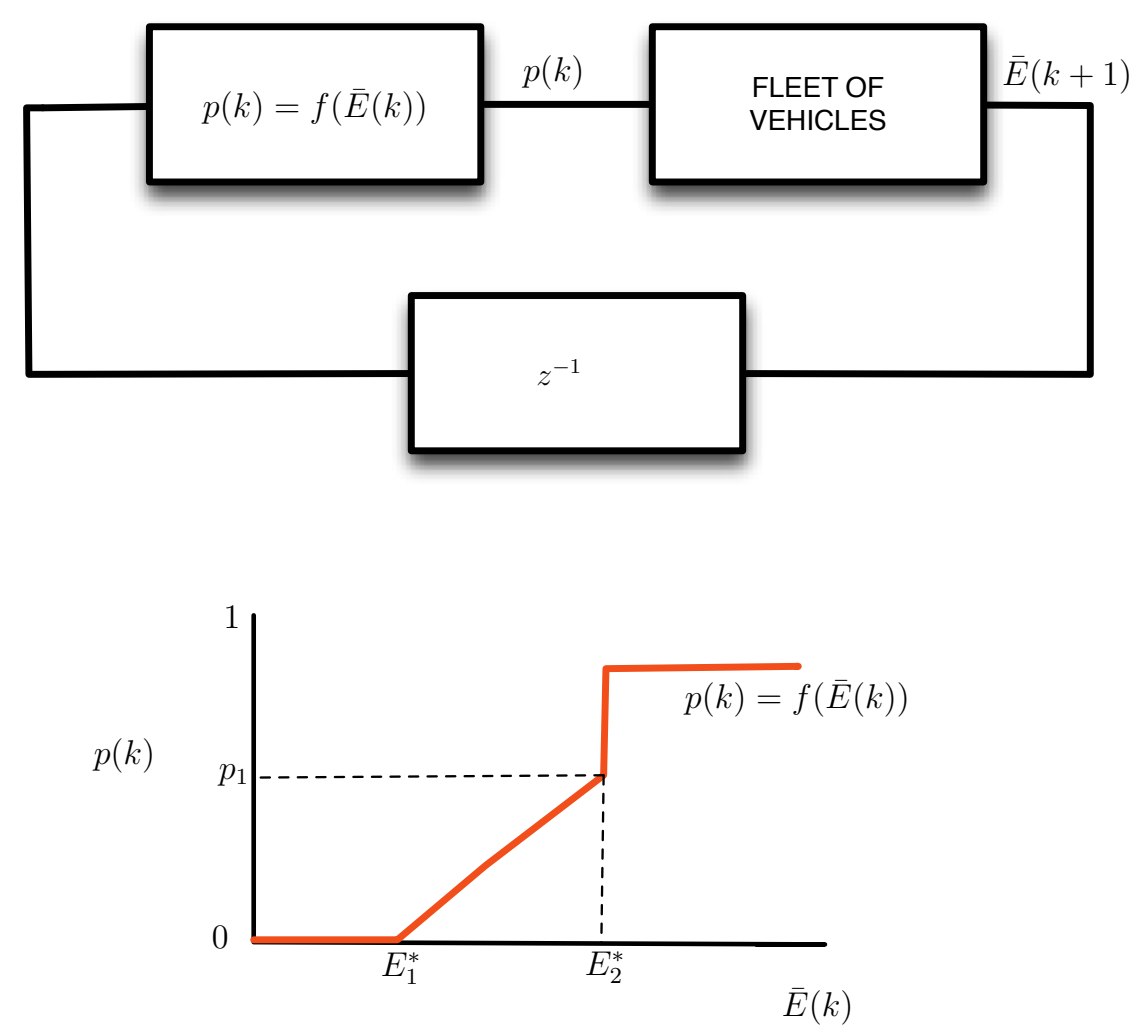

Figure 7: RED feedback loop. RED is the Random Early Detection algorithm used in the internet

$$
p(k+1)=\left\{\begin{array}{lll}
m p(k) & \text { if } & \bar{E}(k)>E_{\text {max }} \\
M p(k) & \text { if } & \bar{E}(k)<E_{\text {min }} \\
p(k) & \text { else } &
\end{array}\right.
$$

This algorithm is designed for quick convergence and fast reaction to changes in the system and disturbances. However its scaling behaviour is not great. The parameters $m$ and $M$ have to be adapted to the specific system. If they are too close to 1 then convergence will be too slow. If they are too far apart from 1 then we will see oscillations. It is also possible to influence this behaviour by choosing a good filter for the emissions. Algorithms like this are being used in the control of computer networks, see for example [36, 37]. 


\section{Simulations}

This section describes the simulation results obtained by implementing the pollution control mechanisms previously described and compares the effects of adopting different utility functions of interest. We employ two different simulation setups, one based on a grid-like network employing the well-known traffic simulator SUMO [26], the other using traffic data deduced from road traffic conditions in Berlin. In both cases we assume that the aggregate levels of pollution can be either measured or estimated. In reality the estimated pollution levels will be a complicated function of both the aggregate pollution levels and the mixing that takes place in a geographical zone. It seems reasonable that this relationship is an increasing function of the aggregate pollution levels, and all our results and algorithms hold in this case as well.

We first describe the grid-based simulation setup in detail. Then we present the results obtained with the different control approaches and for different test scenarios. Then we present results from a more realistic simulation setup to further support the results.

\subsection{Grid simulation setup}

We first present the results for an entirely artificial setup. We simulate traffic in a grid-like transportation network, i.e., where streets intersect orthogonally. Although the investigated grid does not correspond to a particular city, it still reflects the topology of many big cities, that can be found in North America. Traffic is simulated using SUMO and data is sampled every 10 seconds from it. The pollution control strategies are implemented by interfacing Matlab with the SUMO simulator.

For the purpose of the simulations, cars enter at 5 different points in the network and drive along random routes. Over a time interval of 10000 seconds we increase the number of cars in the network until 2000 cars have entered. We then continue the simulation for 6 hours. For the purpose of emissions modeling we assume all cars to be petrol electric hybrid cars with weight below 2.5 Tonnes and with combustion engine capacity larger than $2000 \mathrm{cc}$. We further assume a realistic vehicle mix in terms of their emission standard as described in Section 4.4. The evolution of the number of cars over time in the network is depicted in Figure 8. To better understand the performance of our algorithms, we simulated what happens if we assume, that all cars decide not to use their electric drive at all, or where all cars are just conventional combustion driven vehicles. Figure 9 shows the evolution of emissions over time in this uncontrolled scenario. 


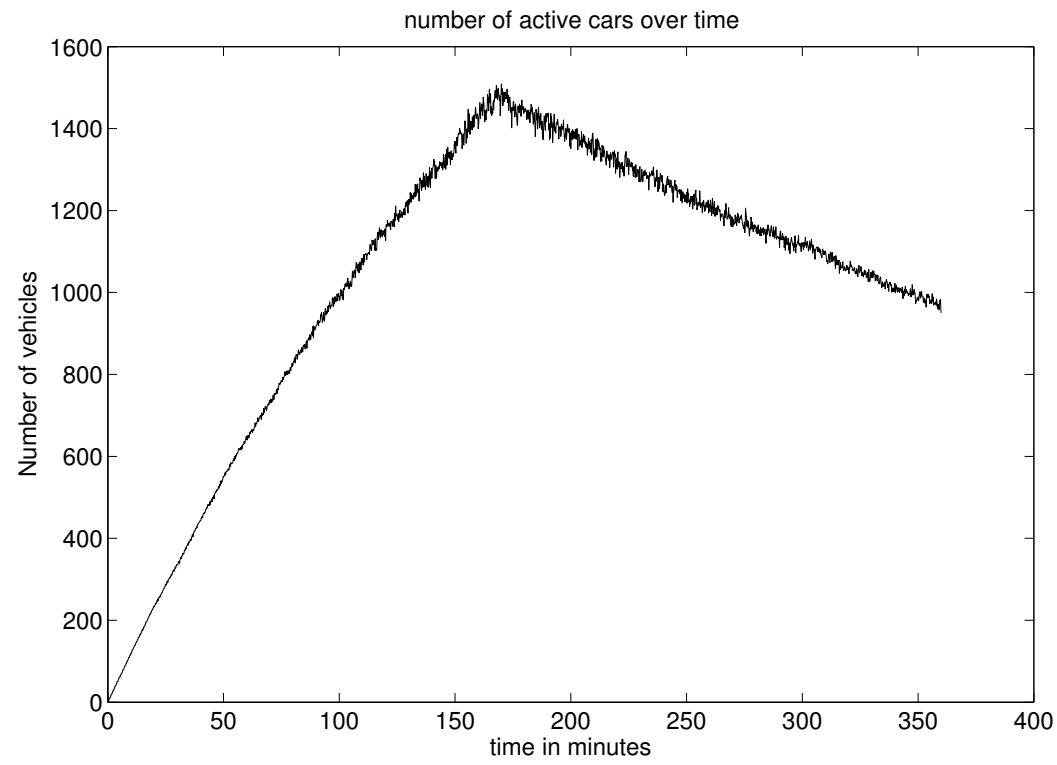

Figure 8: Evolution of the number of vehicles in the network.

\subsection{Different pollution control strategies}

In the first simulation we compare three different control strategies for regulating pollution. We concentrate here on the control of the carbon monoxide emissions, but the same approach can be used for any other pollutant or combinations of pollutants. First we use an MIMD control algorithm, where the aim is to keep the value of the emissions between 40 and 60 grams every minute. Next we used the RED like control algorithm with the same boundary values. Lastly we use an integral control strategy, where we aimed at regulating emissions to the value 50 grams of $\mathrm{CO}$ every minute. The evolution of the emissions and the corresponding evolution of the broadcasted probability in all three scenarios are depicted in Figure 10 Note that the target values we picked for the pollutants were selected arbitrarily but can easily be adapted to safe levels.

Next we repeated the above simulations where we also added an external source of $\mathrm{CO}$ that is active from minute 180 to 270 and contributes 30 grams of $\mathrm{CO}$ per minute during this time. In reality this could for example happen if the wind turns and carries pollution from an industrial area into the city for some time. The results are depicted Figures 11. It can be seen that all three algorithms cope well with this abrupt change.

It can be seen in Figure 9 that if electric drives are not used, the level of 


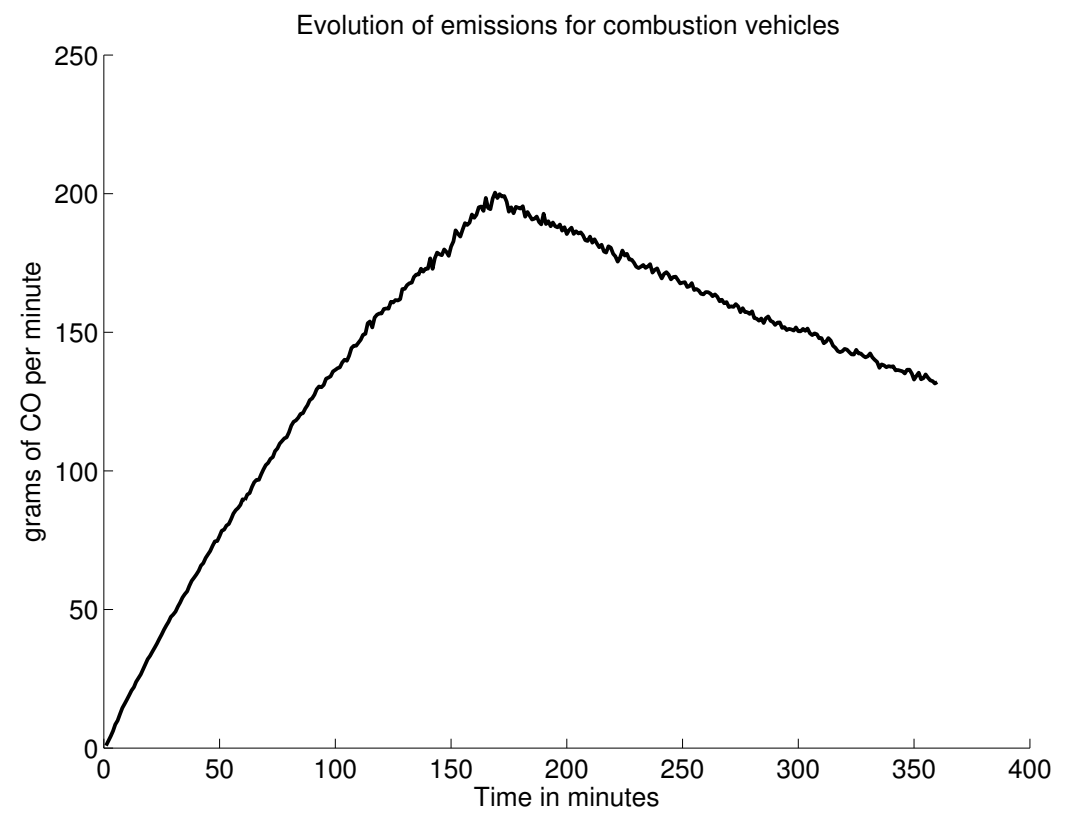

Figure 9: Evolution of the emissions if all vehicles have conventional combustion engines only.

pollution follows a curve that mainly depends on the number of vehicles that are currently traveling in the road network. However, if some of the vehicles travel in EV mode, then the level of emissions does not depend on the number of vehicles anymore, and can be controlled in order to be close to a desired level of pollution, for instance around 50 grams per minute as done here. It can be seen that all three control strategies work well if their respective parameters are tuned to the given scenario. This holds both with and without external pollution. For the remainder of this section we use the integral controller for all simulations.

\subsection{Utility functions}

Figures 10 and 11 clearly emphasise the effectiveness of pollution control strategies in achieving a desired level of pollution. This result was obtained by allowing only some hybrid vehicles to travel in ICE mode, according to a probability distribution that takes into account the distance between the current pollution level and the desired one (i.e., less hybrid vehicles will travel in ICE mode when the pollution is high). In this sense the broadcasted probability can be seen as the desired fraction of cars that travel in ICE mode. Clearly there is a nonunique way in which cars can cooperate to achieve the desired pollution levels 

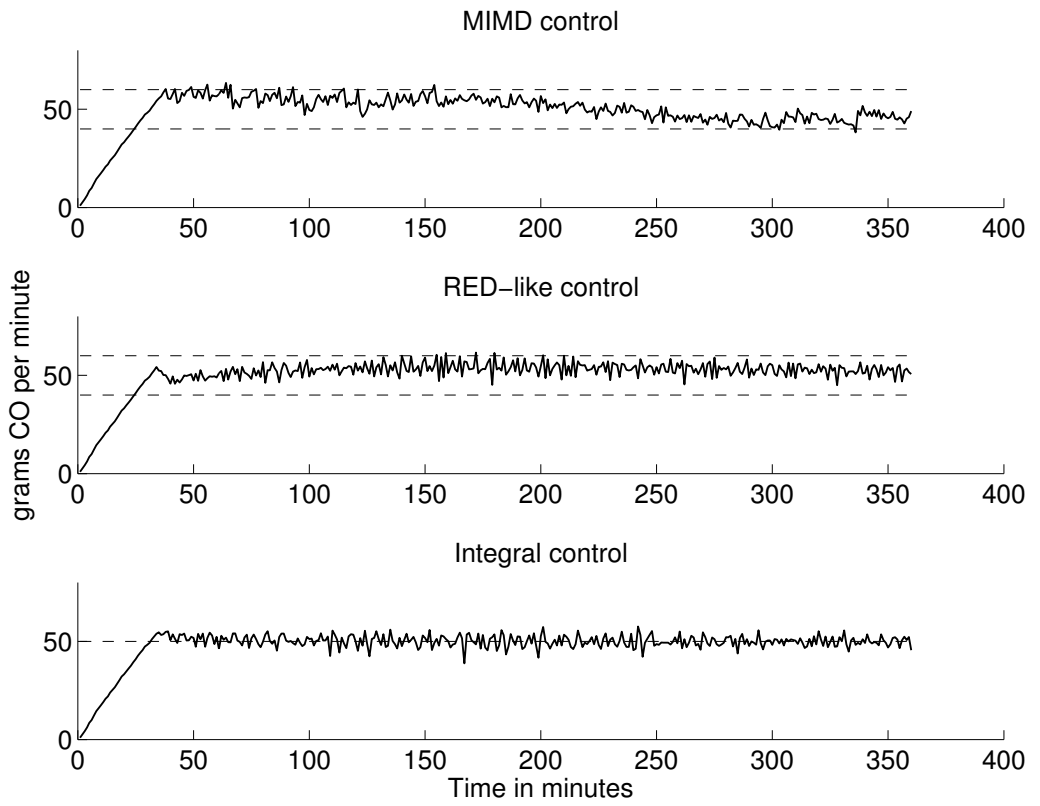

(a)
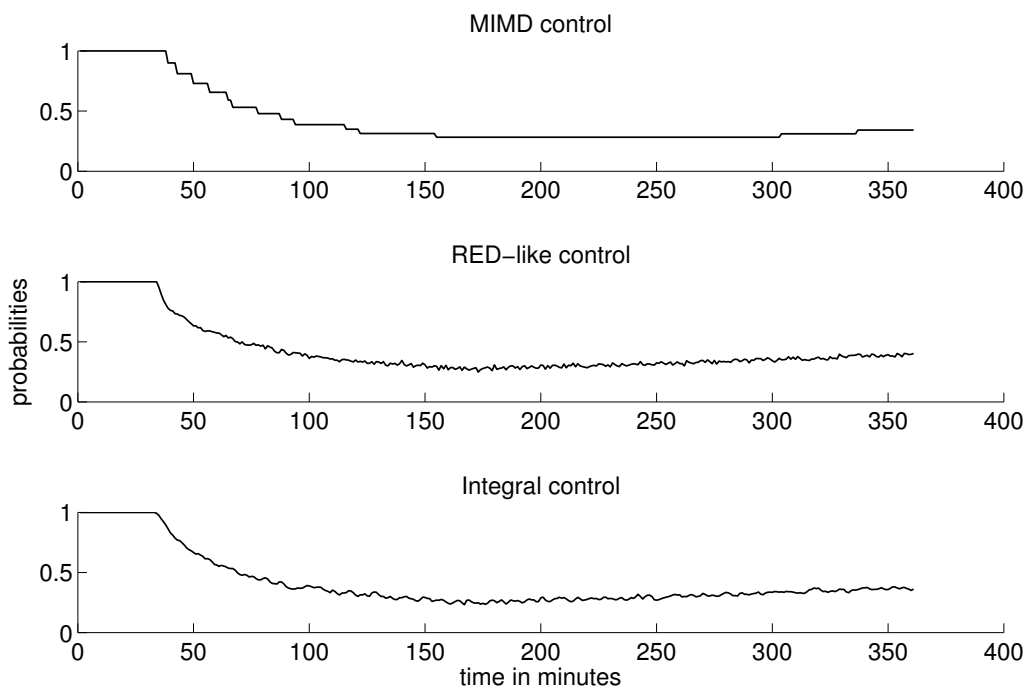

(b)

Figure 10: Comparison of three control approaches. 10a: Evolution of emissions under the three different controllers. 10b: Corresponding probabilities. 


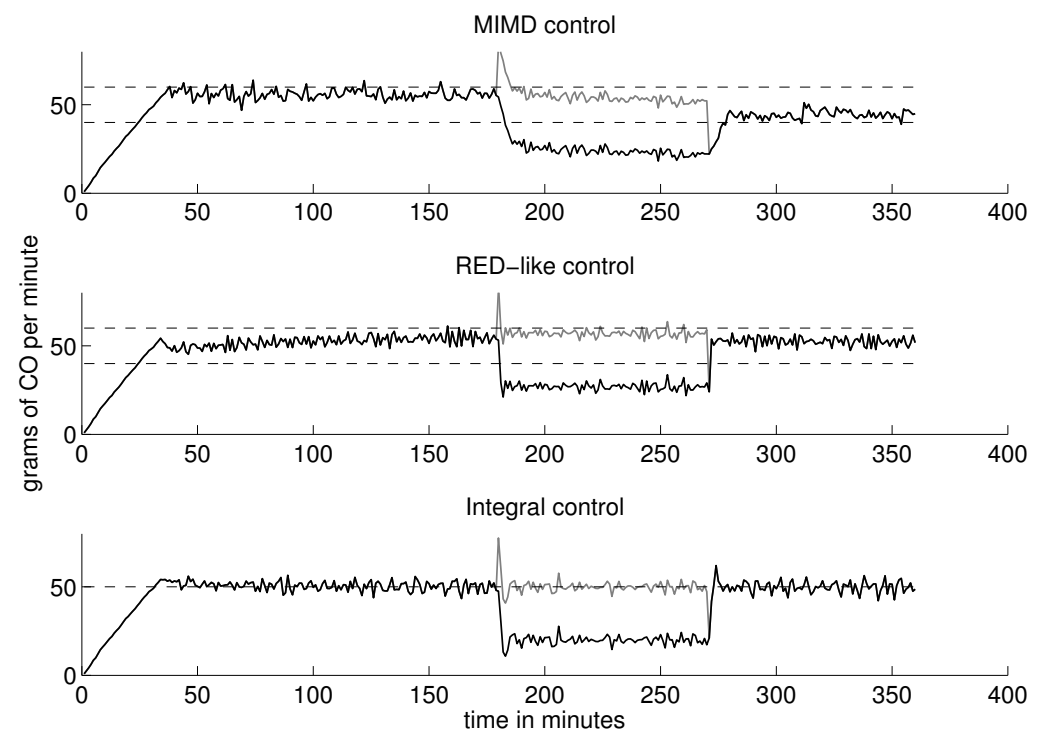

(a)
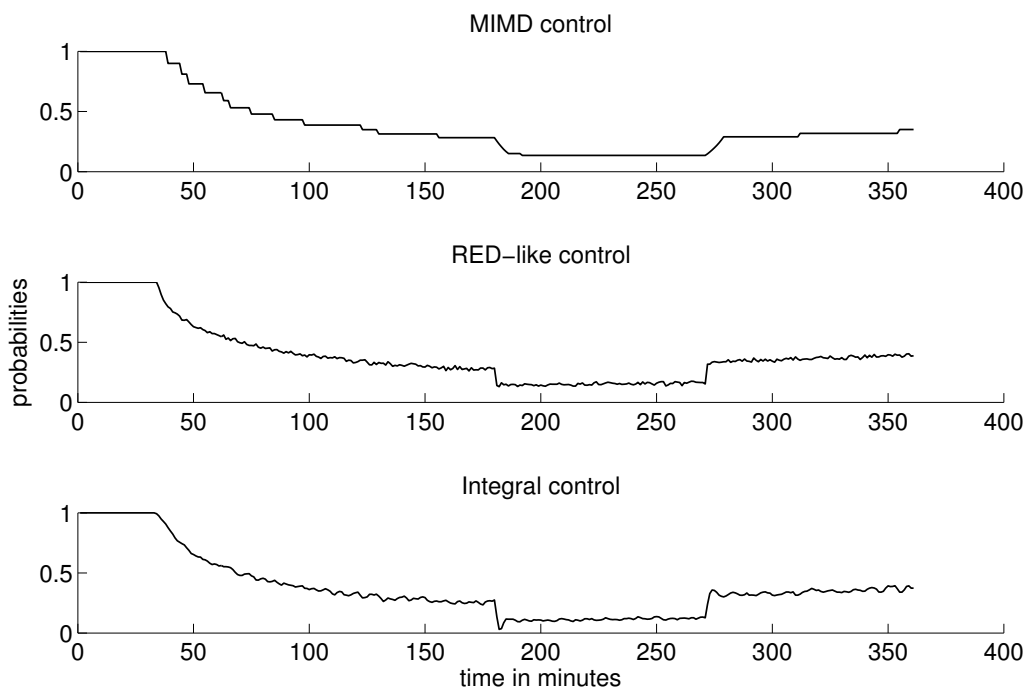

(b)

Figure 11: Comparison of three control approaches in a scenario with an external source of pollution. 11a: Evolution of emissions under the three different controllers. The black line depicts the contribution of the vehicles to the pollution and the grey line shows the aggregate pollution.11b: Corresponding probabilities. 
in a given zone. An exciting aspect of our approach is that this non-uniqueness can be exploited to deliver certain notions of quality of service to individual drivers. For example as we shall see, notions of fairness can be introduced that reflect individual drivers levels of pollution. Such considerations are completely standard in the networking community and are captured by the concept of utility fairness; here, a utility function for each vehicle is used to encapsulate the desired level of fairness between individual drivers. In our context this amounts to tailoring the probability communicated to each vehicle according to the properties of that vehicle (e.g., the type of vehicle or the remaining charge in the battery) or with minimal communication requirements.

\subsubsection{Emission class fairness}

In the first example, we aim at equalising the pollution per vehicle over all simulated emission classes. The idea is that dirty cars will be allowed to travel in ICE mode less often than clean cars. To this end we let each vehicle adjust the probability of traveling in EV mode by considering its own nominal pollution level (i.e., whether it is Euro 1 category rather than Euro 2, or 3 or 4). The overall probability of the $i^{\prime}$ th vehicle to travel in fuel mode then becomes

$$
p_{i}=p * \tilde{p}_{\text {class }}
$$

where $p$ is the broadcasted probability and the same for all vehicles. It depends on the current level of pollution as descried above, and $\tilde{p}_{\text {class }}$ depends on the class of the vehicle. According to this choice of probability we have that dirty vehicles are less likely to be allowed to travel in ICE mode than cleaner vehicles.

As a consequence, Figure 12 shows the adapted probabilities of traveling in fuel mode for each vehicle class. As can be seen, probabilities vary with the level of pollution, but in general dirtier vehicles, i.e., those belonging to class Euro 1, are less likely to be allowed to travel in ICE mode.

Comment : This kind of pollution control strategies based on pollution fairness can in addition be very effective to stimulate car buyers in buying cleaner vehicles, in order to be allowed more time in fuel mode and to spend less time charging their vehicles from the grid.

\subsubsection{Individual pollution fairness}

A further refinement of the above idea is to take into account the actual pollution produced by each vehicle over a time window and to use this as a bases for the utility functions for each vehicle. Clearly cars that spend more time driving in the network tend to produce a larger total amount of pollutants. We can address this issue in one of the two following decentralised ways.

1. Give car $i$ a budget of $C_{i} \geq 0$ units of pollutant it may expend during a trip or during a certain time. Such budget could for example be bought by the driver in a pay-as-you-go fashion or there could be a subscription scheme to do this. The idea then is that the driver can use his ICE complying 


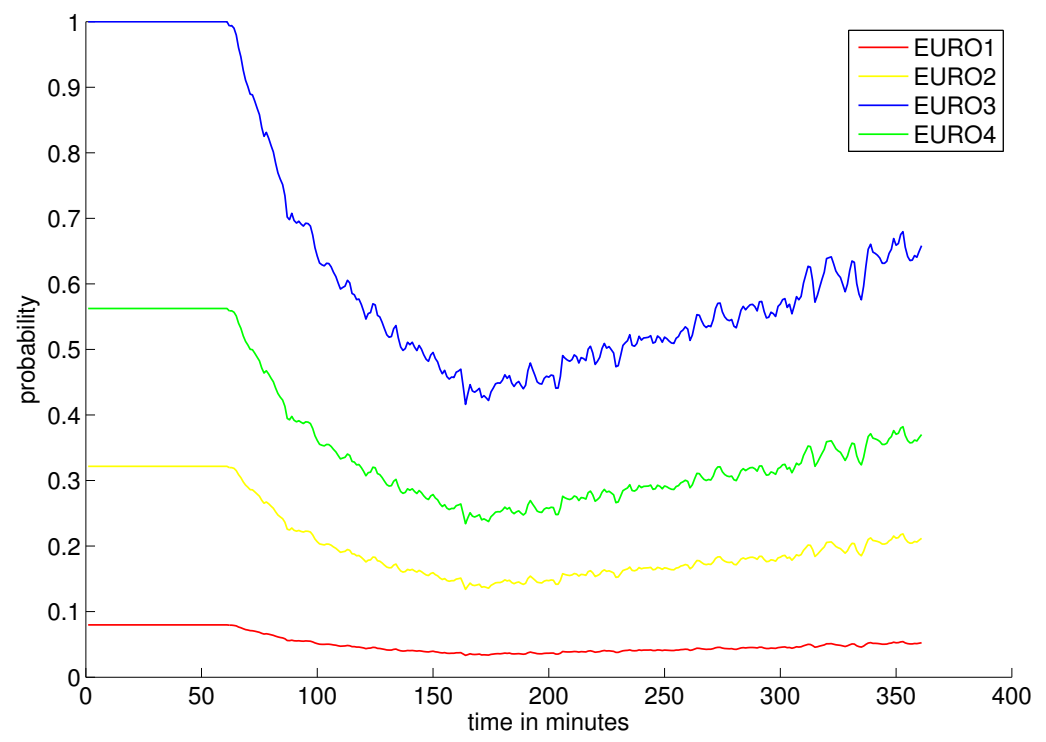

Figure 12: Probabilities of travelling ICE mode depend on the class of the vehicle.

with the pollution control algorithm until he has used all his budget. This can be done in a strict fashion, where the compliance with the algorithm is strict until the budget is used. Another way of doing this is to adapt the broadcasted probability according to

$$
p_{i}=p * \tilde{p}_{\text {pollution }},
$$

where again $p$ is the broadcasted probability and $\tilde{p}_{\text {pollution }}=\frac{\sum_{j=0}^{k} E_{i}(j)}{C_{i}}$, where $E_{i}(j)$ is the amount of pollutant produced by car $i$ at time $j$. In both cases if no budget remains, the driver has to use his electric drive or has to leave the zone or stop the vehicle.

2. The above approach relies on the existence of a budget, for which potentially some infrastructure is required. We present a similar approach that does not require a budget. Let each car calculate a moving average $\bar{E}_{i}(k)$ of its emissions given by $\bar{E}_{i}(k+1)=\lambda E_{i}(k)+(1-\lambda) \bar{E}(k)$. Then a factor $\tilde{p}_{\text {pollution }}$ can be computed according to a common function or a function that depends on the characteristics of the car. The individual probability is then calculated according to

$$
p_{i}=p * \tilde{p}_{\text {pollution }}
$$




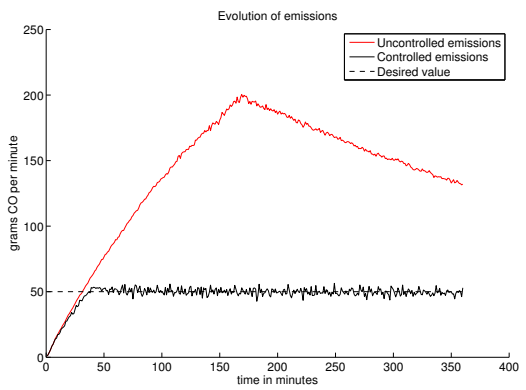

(a)

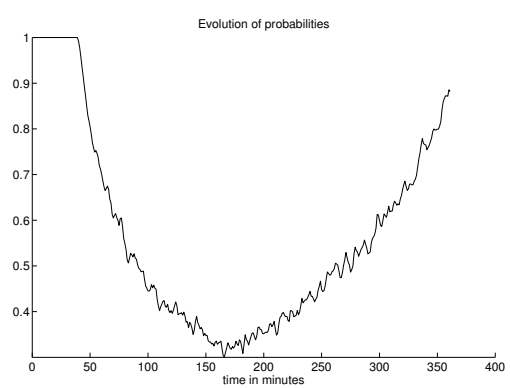

(b)

Figure 13: Pollution control in the individual pollution fairness scenario. 13a: Uncontrolled and controlled pollution. 13b: Evolution of broadcasted probability.

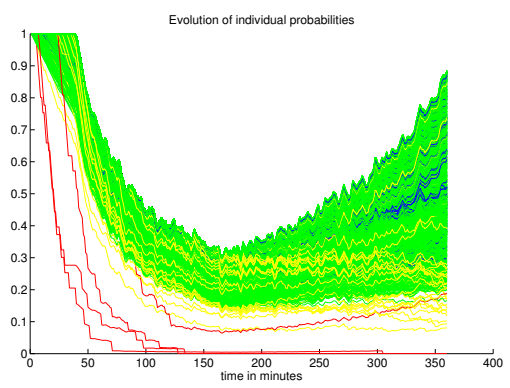

(a)

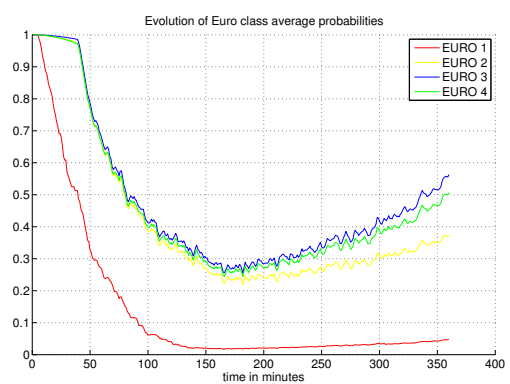

(b)

Figure 14: Evolution of individual probabilities. 14a: Evolution of individual probabilities. 14b: Corresponding ensemble averages per EURO class.

For the simulations presented here we followed the first approach. In the same simulation setup as before we gave each vehicle a budget of 20 grams of $\mathrm{CO}$ to emit over all of its journey. The broadcasted probability was internally adjusted by each vehicle according to Equation (12). In Figure 13a it can be seen that the integral control algorithm still works well in this scenario. The corresponding evolution of the broadcasted probability is depicted in Figure 13b. In Figure 14 we see the evolution of the individually calculated probability used by each vehicle and of the ensemble average probabilities for each emission class over time. Finally in Figure 15 the total amount and the time average amount of pollutants emitted by each car is depicted and compared to the same values in our standard scenario, where the individual pollution fairness was not regarded. 


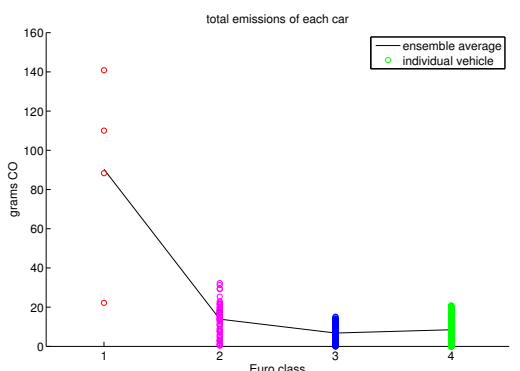

(a)

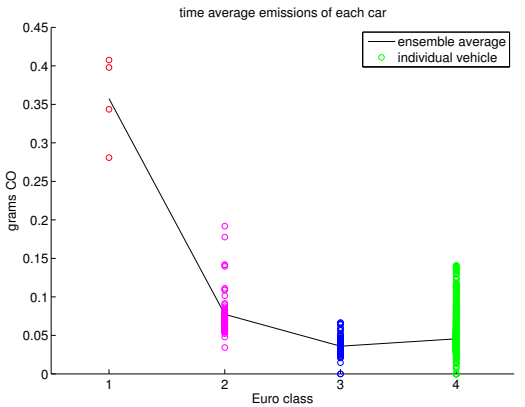

(c)

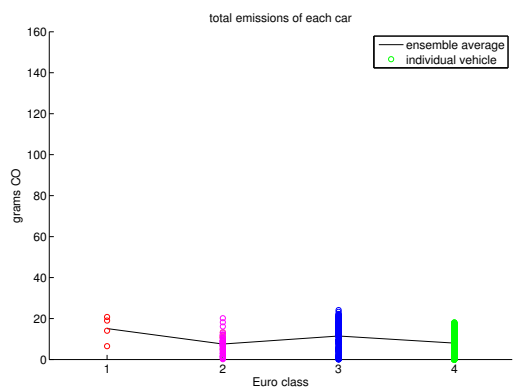

(b)

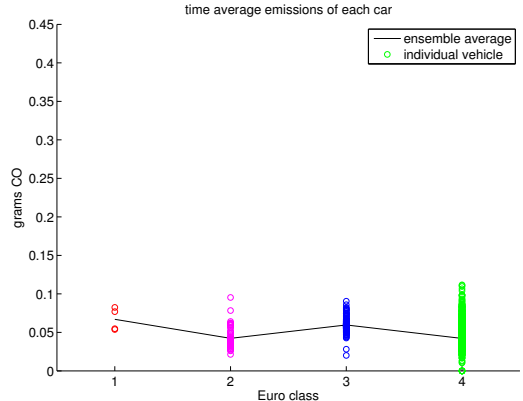

(d)

Figure 15: Per vehicle emissions. For the scenario without the individual pollution fairness, in 15a and 15c we see the total emissions of each car and the time average emissions of each car respectively.15b and $15 \mathrm{~d}$ plot the same information in the scenario with individual pollution fairness. 


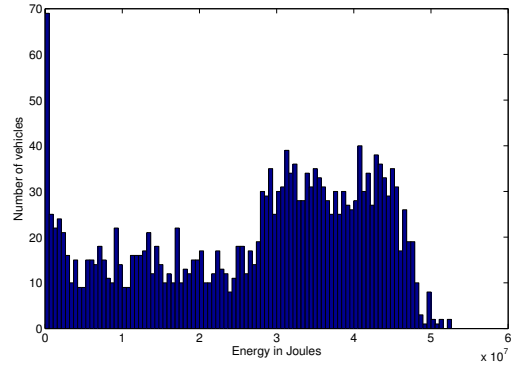

(a)

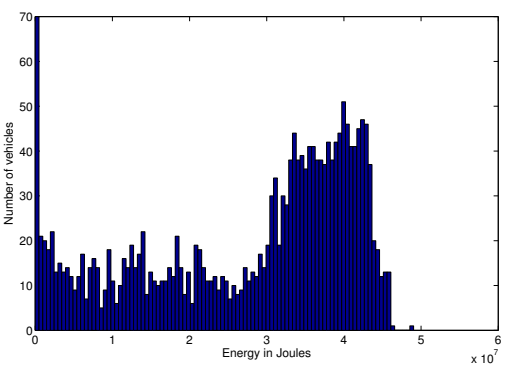

(b)

Figure 16: Histograms of energy used for travelling for each vehicle in the basic scenario without battery fairness $16 \mathrm{a}$ and in the battery fairness scenario $16 \mathrm{~b}$.

\subsubsection{Battery fairness}

In the third example, we assume that each vehicle adjusts the probability of traveling in ICE mode according to the residual level of its battery, so that vehicles that are close to running out of battery can safely drive in fuel mode.

In this case, we adapt the probability of the $i^{\prime}$ th vehicle to travel in ICE mode according to

$$
p_{i}=f\left(p, S O C_{i}\right)
$$

where $S O C_{i}$ is the state of charge of the battery in vehicle $i$ and $f$ : $[0,1] \times \mathbb{R}_{+} \rightarrow[0,1]$ is appropriately computed so that the compliance with the algorithm is strict when the battery is full and $p_{i}$, the probability of car $i$ of driving in ICE mode approaches 1 when the vehicle is running out of battery. In Figure 16 we compare the amount of energy required for driving in the original implementation with the scenario where the broadcasted probability is adjusted in each vehicle according to Equation (14). To be able to do this we gave each simulated vehicle a battery capacity of $5 * 10^{7}$ Joules in order to have enough energy for the pollution control algorithm still to work. Energy requirements were computed according to the model presented in [39]. We consider it undesirable to use more than $4.5 * 10^{7}$ Joules, as this may be harmful for the battery and leave the vehicle without a reasonable reserve of battery charge. Using the battery fairness approach it is possible to reduce the number of vehicles requiring more than the safe amount of energy from 160 to 30.

\subsection{An inner city setup}

As outlined earlier, we want to support the above results by designing a second simulation setup with a greater number of vehicles and with a setup deduced from road traffic conditions in Berlin. Regarding our approach, there are several 
basic input parameters, which are relevant: (1) the traffic density over time, (2) the emission per vehicle and the distribution of vehicle types regarding emission characteristics, and (3) velocities of vehicles. A characteristic distribution of traffic load in Berlin has deduced from [31] and [33]. Here, we focused on passenger cars only. To assess large-scale effects, we refer to the mean number of vehicles per day in Berlin allocated to road network links in Berlin [33]. From here, we selected a number of inner city roads where about 140 thousand vehicles were counted per day. That results - with support of the characteristic distribution of traffic load - in the following numbers of vehicles per hour of day (an artificial and random noise has been included) shown in Figure 17.

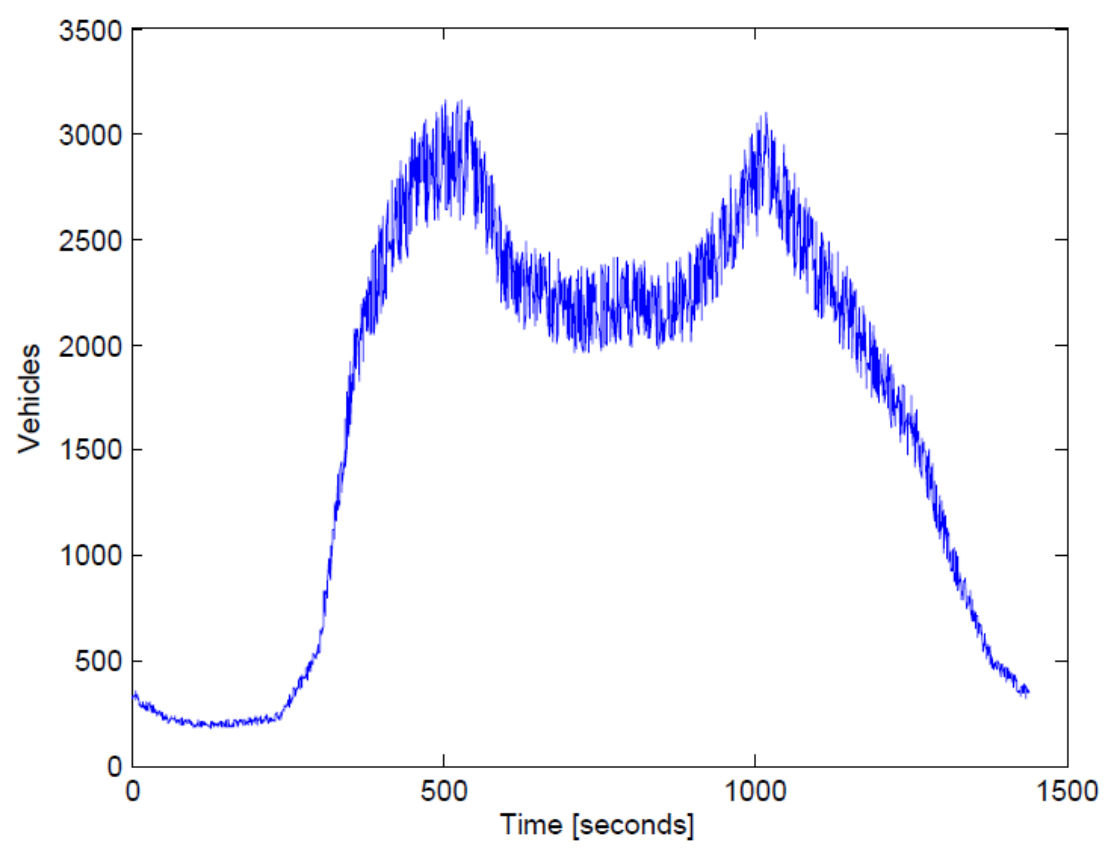

Figure 17: Distribution of vehicles over a day in the city centre.

Regarding the calculation of the individual vehicle's emission, we apply the emission model introduced earlier. We apply the distribution of emission groups in Berlin, which have been instrumented in the context of the Berlin Umweltzone. The emission groups correspond to the European emission standards classes [32]. Different to European emission standard classes, the emission groups counted within the Berlin Umweltzone-context are only five. That is because all European emission classes equal or higher than four are allocated to one group (EURO 4).

The simulation is performed in rounds, which correspond to minutes. That means, that vehicles receive an update for the probability to drive in electric 
mode once a minute. Thus, our simulation runs for one day or 1440 rounds. As outlined earlier, we could distinguish five groups of vehicles in Berlin based on Umweltplaketten distributed in Berlin, with the following shares EURO 4 by $88 \%$, EURO 3 by $9 \%$, EURO 2 by $3 \%$, and EURO 1 by $0.4 \%$. EURO class 0 vehicles are not allowed to enter the Berlin Umweltzone and are thus not considered. We consider equivalent vehicles (regarding weight and fuel type) per group; no heavy vehicles are considered. We assume an even distribution of vehicles velocities between zero and $60 \mathrm{~km} / \mathrm{h}$, which is similar to the Berlin velocity distribution [34].

Based on the setup described above, we employed integral control approach outlined above. Figure 18a shows the aggregated emission over time, with $E^{*}=$ $808 \mathrm{~g} / \mathrm{km}$ and an Integral gain of $K=8 \cdot 10^{-6}$. Figure 18 a shows the evolution of emissions and the desired emission. The Figure 18b depicts the according evolution of probabilities. It is clear that the probability to drive in electric mode must follow the number of emitting vehicles.

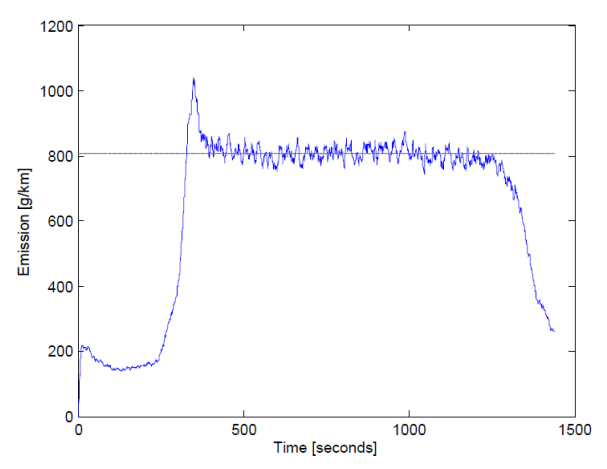

(a)

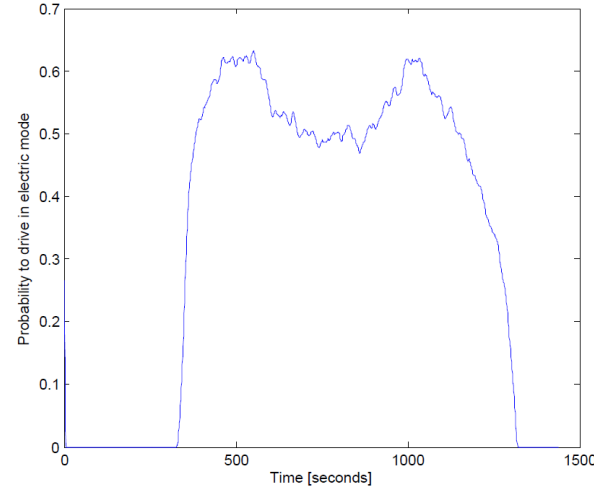

(b)

Figure 18: Evolution of carbon monoxide emission (Figure 18a) with according broadcasted probabilities to drive in electric mode (Figure 18b).

Both figures show that the integral control approach is working as the desired limit of emission is reached also on a larger scale with a characteristic urban setting.

\section{Conclusions and Future Work}

We have presented a novel approach to controlling traffic related emissions in urban scenarios by making use of hybrid vehicles. A specially modified example vehicle was described. We have further given mathematical proof and a number of simulations to showcase the efficacy of our idea. It was shown that our approach is flexible and works in a wide range of scenarios with various objectives 
and controllers.

The ideas presented in this work extend to a number of derivative scenarios. For example it is possible to relax the assumption that all cars are within a small geographic area and instead assume that we regard a company that has a fleet of vehicles, such as a taxi or a delivery company or maybe a public transport organisation. We can give the company an emissions budget that it can then allocate to its vehicles in the same fashion as above. Alternatively the dispatcher can solve an optimisation problem to allocate emission permits to cars based on some utility functions. For example the speed of a vehicle may depend on the drive mode and be higher in ICE mode. Further some tasks may have a higher priority for arriving fast than others. Once the budget is allocated each car can run an additional optimisation to obtain a schedule of when to use the ICE as to minimise its travel time regarding also some hard constraints, i.e. areas in which no polluting is allowed.

As significant fleets of hybrid vehicles are not yet available, we plan to also investigate how similar approaches can be used to control emissions for a fleet of conventional vehicles. For example, when considering an area within a city in which pollution is to be controlled and this area is only accessible through traffic light controlled junctions, it is possible to choose a policy for admission control such that the fraction of green time for traffic lights on streets into the area is proportional to $p$, the broadcasted probability or by controlling speed limits.

We will also be conducting a hardware-in-the-loop test, where our demonstration vehicle will be linked to a traffic simulator while driving in a real road network in order to provide more realistic data to our simulations.

\section{References}

[1] Delivering smarter transportation systems, IBM White Paper, 2007, http://www-935.ibm.com/services/us/igs/pdf/ transport-systems-white-paper.pdf.

[2] Hodgkinson, S., Is your city smart enough, CISCO White Paper, 2011, http://www.cisco.com/web/strategy/docs/ Is-your-city-smart-enough-Ovum-Analyst-Insights.pdf.

[3] http ://www.itif.org/files/2010 - 1 - 27 - ITS - Leadership.pdf

[4] Geng, Y., and Cassandras, C.G., Dynamic resource allocation in urban settings: A smart parking approach, IEEE International Symposium on Computer Aided Control System Design (CACSD), 2011.

[5] Chatterjee, K., Modelling the impacts of transport telematics: Current limitations and future developments, Transport Reviews vol. 19, no. 1, 1999. 
[6] http://www.car2go.com/berlin/en/.

[7] https://www.drive-now.com/lp-promotion1930.

[8] Dirks, S., Constantin Gurdgiev, C. and Keeling, M., Smarter Cities for Smarter Growth, White Paper, IBM Institute for business value, 2010.

[9] Peden, M., McGee, K. and Sharma, G. The Injury Chart Book: A Graphical Overview of the Global Burden of Injuries, World Health Organization, Geneva, 2002.

[10] Position paper, Climate for Transport Change, EU Environmental Agency, http://www. eea. europa.eu/publications/eea-report-2008-1.

[11] FoE (Friends of the Earth) Road Transport, Air Pollution and Health. Briefing Paper, 1997

[12] Cone, T., California Air Pollution Kills More People Than Car Crashes, Study Shows, Article in The Huffington Post, November 13th, 2008.

[13] Knorn, F., Corless, M., and Shorten, R., Results in cooperative control and implicit consensus, International Journal of Control, vol. 84, no. 3, 2011.

[14] Knorn, F., Corless, M. and Shorten, R., A Result on Implicit Consensus with Application to Emissions Control, Proceedings of IEEE CDC, 2011.

[15] Available at http://gis.uba.de/website/umweltzonen/umweltzonen. php.

[16] Available at http://www.brussels.be/artdet.cfm/6357.

[17] Available at http://www.bbc.co.uk/news/uk-politics-12879566.

[18] Shorten, R., Wirth, F., and Leith, D., A positive system model of TCPlike congestion control: asymptotic results, IEEE/ACM Transactions on networking, vol. 14, no. 3, 2006.

[19] Srikant, R., The mathematics of Internet Congestion Control, Systems \& Control:Foundations \& Applications, Birkhaueser, 2003.

[20] Kelly, F.P., Mathematical modelling of the internet, in Proceedings of ICIAM 99, 4th International Congress of Industrial Applied Mathematics, Edinburgh, UK, 1999.

[21] Ehsani, M., Gao, Y., and Emadi, A., Modern electric, hybrid electric and fuel cell vehicles : fundamentals, theory and design, CRC Press, 2010

[22] Gkatzoflias D, Kouridis, Ntziachristos L, Samaras Z, COPERT IV. Computer program to calculate emissions from road transport. User Manual (version 5.0), Published by ETC-ACC (European Topic Centre on Air and Climate Change), 2007. 
[23] Hartenstein, H., and Laberteaux, K., VANET Vehicular applications and internetworking technologies, Wiley, 2010.

[24] Barth M., An F., Younglove T., Scora G., Levine C., Ross M., and Wenzel T., Development of a comprehensive modal emissions model. NCHRP Project 25-11. Final report, 2006.

[25] Highways Agency, Scottish Executive Development Department, Welsh Assembly Government, Department for Regional Development Northern Ireland, Design manual for Roads and Bridges, Volume 11, Section 3, Part 1, Air Quality, Stationary Office, London, 2007.

[26] Krajzewicz, D., Bonert, M., Wagner, P., The open source traffic simulation package SUMO, RoboCup 2006 Infrastructure Simulation Competition, RoboCup 2006, Bremen, Germany, 2006

[27] Budzisz, L., Stanojević, R., Schlote, A., Shorten, R., and Baker, F., On the fair coexistence of loss- and delay-based TCP, IEEE/ACM Transactions on Networking, vol. 19, no. 6, 2011.

[28] Stanojević, R., Shorten, R., and Kellett C., Adaptive tuning of Drop-Tail buffers for reducing queueing delays. IEEE Comms Letters, vol. 10, no. 7, 2006.

[29] Kellett, C., Shorten, R., and Leith, D., Sizing internet buffers, Active queue management, and the Lure problem. Proceedings of IEEE Conference on Decision and Control, 2006.

[30] Shorten, R., Wirth, F., Mason, O., Wulff, K., and King, C., Stability Criteria for Switched and Hybrid Systems, SIAM Rev., 49(4), pp. 545-592, November 2007.

[31] Senatsverwaltung für Stadtentwicklung und Umweltschutz Berlin (Ed.), Umweltaltlas Berlin, Karte 07.01 Verkehrsmengen (1995), available online at URL:http://www.stadtentwicklung.berlin.de/umwelt/umweltatlas/ d701_03.htm.

[32] GEMB Gesellschaft für Emissionsmanagement und Beratung GmbH, Informationsportal zur Deutschen Umweltplakette, available at http://www . umwelt-plakette.de.

[33] Senatsverwaltung für Stadtentwicklung und Umweltschutz Berlin (Ed.), Umweltaltlas Berlin, Karte 07.01 Verkehrsmengen (2011), available at http://www.stadtentwicklung.berlin.de/umwelt/umweltatlas/ karten/pdf/07_01_2009_mit_strname.pdf.

[34] Steven, H., Comparison of different European databases with respect to road category and time periods (on peak, off peak, weekend), available at http://www.unece.org/fileadmin/DAM/trans/doc/ 2010/wp29grpe/WLTP-DHC-03-04e.ppt. 
[35] Boulter, P.G., Barlow, T.J., McCrae, I.S., Emission factors 2009: Report 3: exhaust emission factors for road vehicles in the United Kingdom, Published project report PPR356, TRL Limited, 2009.

[36] Stanojević, R., Router-based algorithms for improving internet quality of service, $\mathrm{PhD}$ thesis, National University of Ireland Maynooth, 2007.

[37] Feng, W., Kandlur, D., Saha, D., and Shin. K., A Self-Configuring RED Gateway, Proceedings of INFOCOM, New York, NY, USA, 1999.

[38] Floyd, S., and Jacobson, V., Random Early Detection gateways for Congestion Avoidance, IEEE/ACM Transactions on Networking, vol. 1, no. 4, pp. 397-413, 1993.

[39] Schlote, A., Crisostomi, E., Kirkland, S., Shorten, R., Traffic Modelling Framework for Electric Vehicles, International Journal of Control, Vol. 85, no. 7, pp. 880-897, 2012. 